\title{
New advances in data, information, and knowledge in the Internet of Things
}

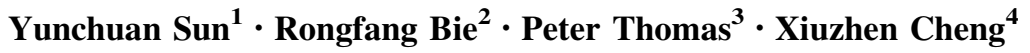

Published online: 23 August 2016

(C) Springer-Verlag London 2016

As the infrastructure of the information society, the Internet of Things (IoT) has created opportunities for more direct integration of the physical world into computer-based systems, resulting in improved efficiency, accuracy, and economic benefit. We are still a long way away from a truly connected world. Some major challenges still exist, such as shared standards and infrastructure, data control and access, and data security. These challenges are the focus for the International Conference on Identification, Information and Knowledge in the Internet of Things (IIKI), where international experts all over the world discuss state-of-the-art solutions and current trends related to various issues in the IoT. This theme issue is in collaboration with the international event the International Conference on Identification, Information, and Knowledge in The Internet of Things (IIKI 2015, http://business.bnu.edu. cn/IIKI2015/) held on October 22-23, 2016, Beijing, China. Fifteen papers were accepted for this issue.

Yunchuan Sun

yunch@bnu.edu.cn

Rongfang Bie

rfbie@bnu.edu.cn

Peter Thomas

peter.thomas@manifesto-group.com

Xiuzhen Cheng

cheng@gwu.edu

1 Business School, Beijing Normal University, Xin Jie Kou Wai Street 19, Beijing 100875, China

2 College of Information Science and Technology, Beijing Normal University, Beijing, China

3 Brunel University, London, UK

4 The George Washington University, 801 22nd St. NW, Suite 704, Washington, DC 20052, USA
The paper [1] entitled "Validation of Smartphone Gyroscopes for Mobile Biofeedback Applications" aims to validate smartphone gyroscopes for angular tracking in mobile biofeedback applications. The validation method includes measurements of angular motion performed concurrently by the smartphone gyroscope and the professional optical tracking system serving as the reference. The comparison of measurement results shows that the inaccuracies of a calibrated smartphone gyroscope for various movements are between $0.42^{\circ}$ and $1.15^{\circ}$, leading to a conclusion that smartphone gyroscopes are sufficiently accurate for angular motion tracking in mobile biofeedback applications.

The paper [2] entitled "Interference Coordination based on Random Fractional-Spectrum Reuse in Femtocells towards Internet of Things" proposes an inter-femtocell interference coordination scheme to enable random and fractional reuse of frequency resources in a 3D in-building scenario. Specifically, they consider the regular femtocell deployment, where all the femtocells are divided into two groups and two neighboring femtocells will be classified into different groups. The simulation results show that the proposed schemes could obtain the larger system average rate and edge user performance compared with the baseline schemes.

The paper [3] entitled "A Two-time-scale Load Balancing Framework for Minimizing Electricity Bills of Internet Data Centers" formulates an electricity bills minimization problem and proposes a novel two-time-scale load balancing framework TLB to solve it. TLB transforms the primal optimization problem into a typical mixed-integer linear programming (MILP) problem and solves it to finally obtain the optimal scheduling policy including the open server number as well as the request routing policy. As an application instance of TLB, a two-time-scale load 
balancing algorithm TLB-ARMA is also designed for the experimental scenario. Evaluation results based on real-life traces show that TLB-ARMA is able to reduce the total electricity bills by as much as $12.58 \%$ compared with the hourly executed GGLB without incurring the costly repeated on-off switching of servers.

The paper [4] entitled "A proportional Fairness Scheduling for Wireless Sensor Networks" describes a packet scheduling algorithm for wireless sensor networks (WSNs) that meets the proportional fairness principle. Based on the weighted round-robin (WRR) strategy, the proposed scheduling algorithm allocates a different service quota to different traffic according to the average packet arrival rate. The proposed scheduling algorithm is tested in a WSN and was found to guarantee the proportional fairness of the average packet delivery delay when this is used as the performance metric and to realize proportional fairness in the average packet loss ratio when all the queues are overflowing and the average packet loss ratio is used as the performance metric.

The paper [5] entitled "A Novel Contention-on-Demand Design for WiFi Hotspots" proposes a novel contentionon-demand (CoD) MAC scheme to address the problem of maximizing the channel utilization while providing satisfactory user experiences. The $\mathrm{CoD}$ scheme consists of a fixed-CW algorithm, a dynamic-CW algorithm, and an admission control rule. An asymptotic analysis was performed to develop a simple and practical admission control rule for homogeneous and heterogeneous traffic.

The paper [6] entitled "Retrieving the Maximal TimeBounded Positive Influence Set from Social Networks" studies the problem of selecting the optimal individual subset to diffuse the positive influence when time is bounded. They prove that such a problem is NP-hard and propose a heuristic algorithm based on greedy strategy. The experimental results on both simulation and real-world social networks based on the trace data in Shanghai show that the proposed algorithm outperforms the existing algorithms significantly, especially when the network structure is sparse.

The paper [7] entitled "Automatically Constructing Course Dependence Graph based on Association Semantic Link Model" proposes an approach utilizing association semantic link model for automatically constructing course dependence graph. The advantage of the proposed approach is that it promotes the automation of constructing course dependence graph, defending its objectivity and getting the service of the course dependence graph smarter. The experiments show that the proposed approach has rationality and validity.

The paper [8] entitled "Building Text-based Temporally Linked Event Network for Scientific Big Data Analytics" surveys existing research work on text-based event temporal resolution and reasoning including identification of events, temporal information resolutions of events in English and Chinese texts, the rule-based temporal relation reasoning between events and the temporal representations. They point out the shortcomings of existing research work and provide an argument about future research work for advancing the identification of events, the establishing of temporal relations and reasoning of temporal relations.

The paper [9] entitled "Connected Dominating Set Construction in Cognitive Radio Networks" studies the connected dominating set (CDS) construction in cognitive radio networks (CRNs) and proposes a three-phase centralized algorithm and a distributed algorithm, which is inspired by the success of constructing a CDS as a virtual backbone realizing efficient topology control in traditional wireless networks.

The paper [10] entitled "An Energy-Efficient SourceAnonymity Protocol in Surveillance Systems" proposes the dynamic optimal mixring-based source-location anonymity protocol, DORing. The simulation results demonstrate that DORing is very efficient in balancing energy consumption and transmission latency and can significantly prolong survival period of the network and ensure security as well as latency to satisfy the packets' requirements.

The paper [11] entitled "Adaptive fuzzy clustering by fast search and find of density peaks" proposes a fuzzy method based on clustering by fast search and find of density peaks (CFSFDP) for adaptively selecting the cluster centers effectively, which uses the fuzzy rules, based on the density assumption for the selection of cluster centers. The experimental results on nine synthetic clustering datasets and the comparison of the stateof-the-art methods validate the robustness and effectiveness of proposed fuzzy-CFSFDP method.

The paper [12] entitled "QoS4IVSaaS: A QoS Management Framework For Intelligent Video Surveillance As A Service" proposes QoS4IVSaaS, a QoS framework to make computing resources highly available. The framework takes multiple metrics into account and proposes a model selection algorithm to choose the model that achieves the best performance under various error indicators. Evaluation results show that the proposed framework can successfully ensure QoS by dynamically scheduling computing resources.

The paper [13] entitled "Node Localization Algorithm for Wireless Sensor Networks Using Compressive Sensing Theory" proposes an indoor localization algorithm based on dynamic measurement compressive sensing for wireless sensor network. The algorithm builds a potential area that possesses the independent features with a bounding-box method, which can decrease the number of meshing and reduce the dimension of measurement matrix. The simulation results indicate that the proposed algorithm can 
reduce the time complexity, while also maintaining localization accuracy and localization efficiency.

The paper [14] entitled "A Framework for Physiological Indicators of Flow in VR Games: Construction and Preliminary Evaluation" constructs a physiological evaluation model to evaluate flow in virtual reality (VR) game. The evaluation model consists of 5 first-level indicators and their respective second-level indicators. An empirical experiment is conducted to test the effectiveness of partial indicators to predict flow experience. Most results support the model and reveal that heart rate (HR), interbeat interval (IBI), heart rate variability (HRV), low-frequency HRV (LF-HRV), high-frequency HRV (HF-HRV), and respiratory rate (RR) are all effective indicators in predicting flow experience.

The paper [15] entitled "An Energy Efficient PrivacyPreserving Content Sharing Scheme in Mobile Social Networks" proposes a Publish-subscribe System with Secure Proxy Decryption (PSSPD) in mobile social networks. First, an effective self-contained privacy-preserving access control method is introduced to protect the confidentiality of the content and the credentials of users; after that, a secure proxy decryption mechanism is proposed to reduce the heavy burdens of energy consumption on performing ciphertext decryption at end users. The experimental results demonstrate the efficiency and privacy preservation effectiveness of PSSPD.

We would like to appreciate all authors for their valuable contributions to this theme issue. Our gratitude also goes to the reviewers for their careful work and thoughtful suggestions that have helped to improve the manuscripts substantially.

\section{References}

1. Umek A, Kos A (2016) Validation of smartphone gyroscopes for mobile biofeedback applications. Pers Ubiquit Comput. doi:10. 1007/s00779-016-0946-4
2. Zhang G, Chu M, Li J (2016) Interference coordination based on random fractional-spectrum reuse in femtocells towards internet of things. Pers Ubiquit Comput. doi:10.1007/s00779-016-0947-3

3. Dou H, Qi Y, Wei W, Song H (2016) A two-time-scale load balancing framework for minimizing electricity bills of internet data centers. Pers Ubiquit Comput. doi:10.1007/s00779-0160941-9

4. Huang J, Bi J (2016) A proportional fairness scheduling for wireless sensor networks. Pers Ubiquit Comput. doi:10.1007/ s00779-016-0948-2

5. Feng L, Yu J, Cheng X, Atiquzzaman M (2016) A novel contention-on-demand design for WiFi hotspots. Pers Ubiquit Comput. doi:10.1007/s00779-016-0942-8

6. Shi T, Cheng S, Cai Z, Li Y, Li J (2016) Retrieving the maximal time-bounded positive influence set from social networks. Pers Ubiquit Comput. doi:10.1007/s00779-016-0943-7

7. Zhou P, Liu J, Yang X, Cui X, Chang L, Zhang S (2016) Automatically constructing course dependence graph based on association semantic link model. Pers Ubiquit Comput. doi:10. 1007/s00779-016-0950-8

8. Zhang J, Yao C, Sun Y, Fang Z (2016) Building text-based temporally linked event network for scientific big data analytics. Pers Ubiquit Comput. doi:10.1007/s00779-016-0940-x

9. Yu J, Li W, Cheng X, Atiquzzaman M, Wang H, Feng L (2016) Connected dominating set construction in cognitive radio networks. Pers Ubiquit Comput. doi:10.1007/s00779-016-0944-6

10. Niu X, Zhang Y, Yao Y, Chen X, Jornet JM, Liu J (2016) An energy-efficient source-anonymity protocol in surveillance systems. Pers Ubiquit Comput. doi:10.1007/s00779-016-0949-1

11. Bie R, Mehmood R, Ruan S, Sun Y, Dawood H (2016) Adaptive fuzzy clustering by fast search and find of density peaks. Pers Ubiquit Comput. doi:10.1007/s00779-016-0954-4

12. Zhang W, Duan P, Xie X, Xia F, Lu Q, Liu X, Zhou J (2016) QoS4IVSaaS: a QoS management framework for intelligent video surveillance as a service. Pers Ubiquit Comput. doi:10. 1007/s00779-016-0945-5

13. Wei Y, Li W, Chen T (2016) Node localization algorithm for wireless sensor networks using compressive sensing theory. Pers Ubiquit Comput. doi:10.1007/s00779-016-0951-7

14. Bian Y, Yang C, Gao F, Li H, Zhou S, Li H, Sun X, Meng X (2016) A framework for physiological indicators of flow in VR games: construction and preliminary evaluation. Pers Ubiquit Comput. doi:10.1007/s00779-016-0953-5

15. He Z, Cai Z, Han Q, Tong W, Sun L, Li Y (2016) An energy efficient privacy-preserving content sharing scheme in mobile social networks. Pers Ubiquit Comput. doi:10.1007/s00779-0160952-6 